\title{
Progressive hypoxia causes reversible left ventricular D- shaping in an experimental porcine model
}

\section{Ringgaard V.K ${ }^{1}$, Sorensen A.H' ${ }^{1}$, Wemmelund K.B ${ }^{1}$., Sloth $\mathrm{E}^{1}$. Juhl-Olsen $\mathbf{P}^{2}$.}

1 Department of anaesthesiology and intensive care medicine, Aarhus University Hospital

2 Department of anaesthesiology, Randers Regional Hospital

\section{Background:}

In POC echocardiography, a pulmonary embolism is characterized by a D-shaping of the left ventricle. The specificity of this characteristic has recently been challenged, as hypoxia induced by acute asphyxia resulted in D-shaping of the left ventricle. However, decompensation of respiratory distress most often manifests as progressive hypoventilation.

Therefore, we aimed to evaluate the left ventricular ultrasonographic appearance of progressive hypoventilation using a porcine model.

We hypothesized that progressive hypoventilation would lead to a gradual D-shaping of the left ventricle.

\section{Results:}

Left ventricular eccentricity index (LV-EI), an ultrasonographic index of D-shaping, increased from 1.1 (1.0-1.1) at baseline to 1.4 (1.3-1.4) three minutes into the final intervention $(P<0.001)$. After two minutes of respiratory resuscitation LV-El returned to baseline (1.1 (1.1-1.2), $\mathrm{P}=0.093)$ and stayed at this level $(\mathrm{P}=0.348)$ (fig 1)
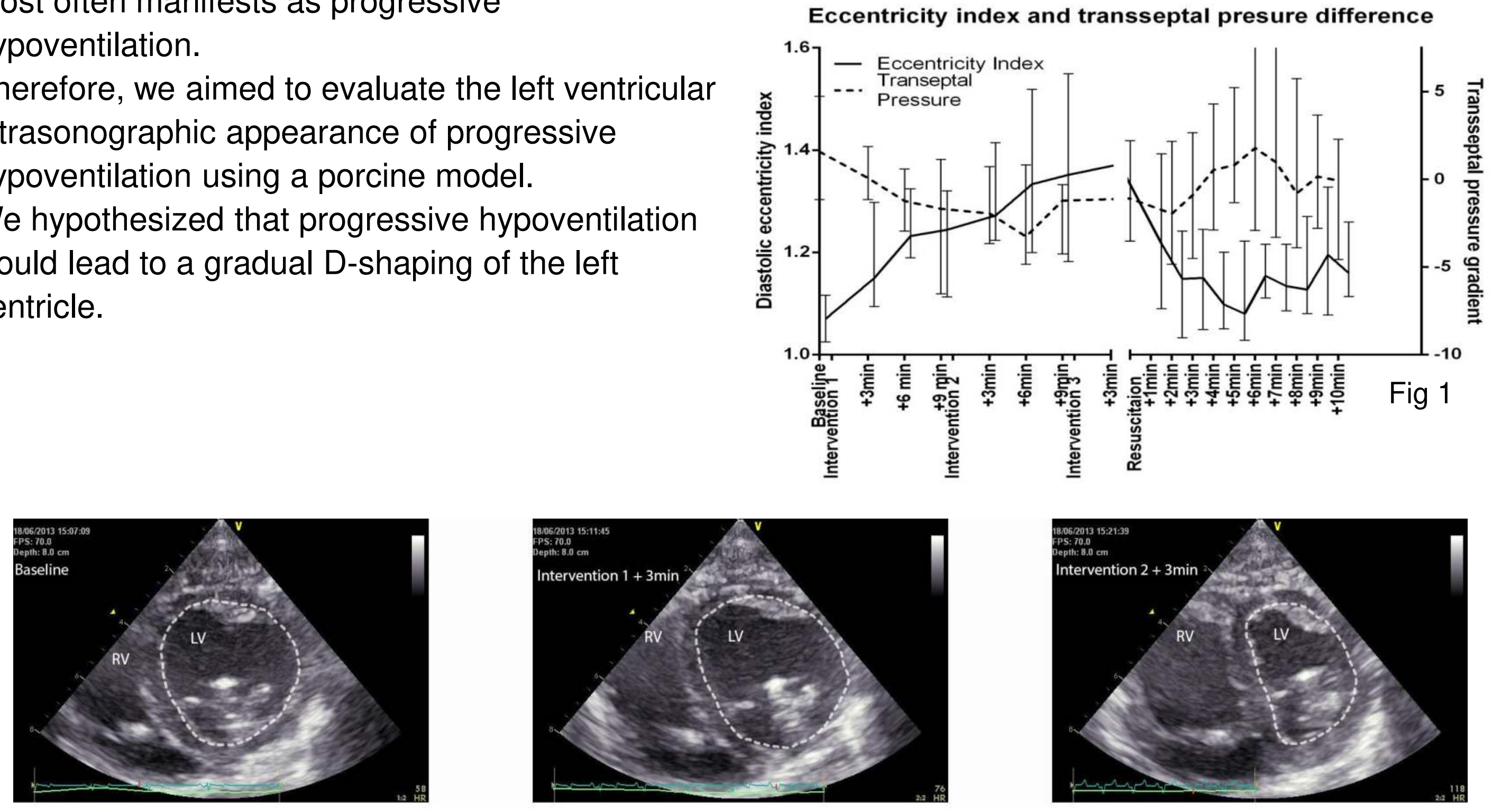

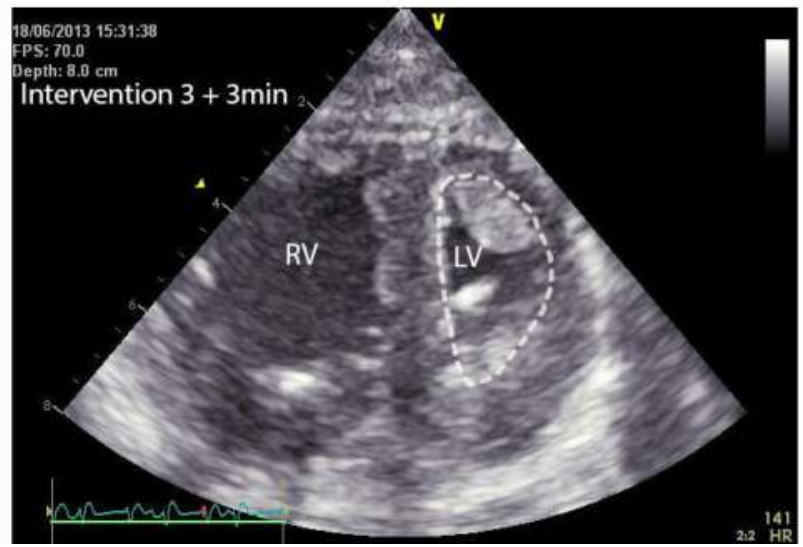

Materials and Methods:

The Danish Inspectorate of Animal Experimentation approved the study (authorization number: 2013-152934-00765). Fifteen piglets (intervention $n=10$ control $\mathrm{n}=5$ ) were included in the study. The intervention piglets underwent three ten-minute periods of graded hypoventilation before respiratory resuscitation was initiated. Parasternal short axis view cineloops were stored and arterial blood samples were drawn after 3, 6 and 9 minutes at each level of hypoventilation. During resuscitation, cineloops were stored every minute and arterial blood samples drawn every second minute.

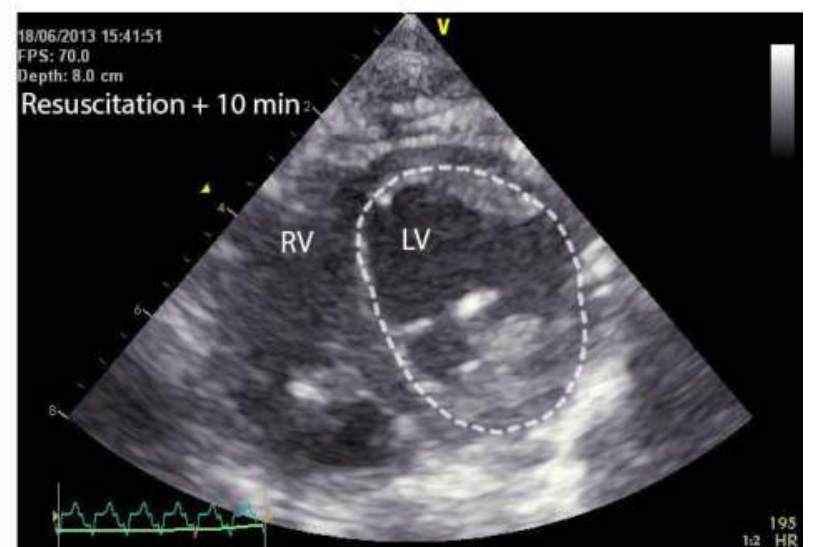

Fig 2 Ultrasonograpic images

\section{Discussion and conclusion:}

Progressive hypoventilations yields a reversible echocardiographic image(Fig 2), which to many clinicians is synonymous with pulmonary embolism. This questions signs of right ventricular overload as a specific rule-in diagnostic, and may make repeated echocardiography after reoxygenation an important differential diagnostic tool.

Progressive hypoventilation caused reversible D-shaping of the left ventricle and poses a differential diagnosis to pulmonary embolism. 